\title{
Metric perturbations in two-field inflation
}

\author{
Juan García-Bellido and David Wands \\ Astronomy Centre, School of Mathematical and Physical Sciences, \\ University of Sussex, Brighton BN1 9QH, U.K.
}

(August 9, 2018)

\begin{abstract}
We study the metric perturbations produced during inflation in models with two scalar fields evolving simultaneously. In particular, we emphasize how the large-scale curvature perturbation $\zeta$ on fixed energy density hypersurfaces may not be conserved in general for multiple field inflation due to the presence of entropy as well as adiabatic fluctuations. We show that the usual method of solving the linearized perturbation equations is equivalent to the recently proposed analysis of Sasaki and Stewart in terms of the perturbed expansion along neighboring trajectories in field-space. In the case of a separable potential it is possible to compute in the slow-roll approximation the spectrum of density perturbations and gravitational waves at the end of inflation. In general there is an inequality between the ratio of tensor to scalar perturbations and the tilt of the gravitational wave spectrum, which becomes an equality when only adiabatic perturbations are possible and $\zeta$ is conserved.
\end{abstract}

98.80.Cq Preprint SUSSEX-AST 95/11-4, IEM-FT-119/95, astro-ph/9511029

\section{INTRODUCTION}

Inflation is the only known mechanism that solves the horizon and homogeneity problems of hot big bang cosmology [1]. However, the main observational prediction of inflationary models is the spectrum of density and gravitational wave perturbations they produce. Observations of temperature anisotropies in the microwave background, strictly speaking only provide an upper limit on the amplitude of such perturbations, and could in principle be produced by some other source of inhomogeneities. Nonetheless, the apparently Gaussian and nearly scaleinvariant nature of the observed perturbations are natural properties of those produced by quantum fluctuations of the inflaton field during inflation. If inflation is indeed responsible for the observed anisotropies of the microwave background and the initial curvature perturbations from which galaxies formed, then the amplitude over a limited range is already constrained by observations [2, 4]. In future both the range and precision of these constraints promise to improve considerably and so it will be increasingly important to fully understand the predictions made by the inflationary paradigm and the robustness of these predictions.

Until comparatively recently it was often stated that inflation predicts a scale-invariant Harrison-Zel'dovich spectrum of density perturbations with a negligible amplitude of gravitational waves. In fact, both the tilt of the spectrum and the relative contribution of gravitational waves to the microwave background anisotropies are model-dependent quantities [2]. In the conventional model of inflation driven by the potential energy density of a single slowly-rolling scalar field, the tilt and the ratio of tensor (gravitational wave) to scalar (density) perturbations in the microwave background can be determined by the slow-roll parameters which describe the slope and curvature of the potential as the perturbations cross out- side the horizon during inflation 5 . Scalar curvature perturbations $\zeta$ can be understood as originating from quantum fluctuations of the inflaton field that perturb the time it takes to end inflation [6], $\zeta=H \delta t=H \delta \phi / \dot{\phi}$. Our ability to determine the linear perturbation at late times solely in terms of the parameters at horizon crossing depends on the constancy of $\zeta$ on scales far outside the horizon. The value of $\zeta$ when a given comoving scale leaves the horizon during inflation can then be equated with that at re-entry during the radiation- or dust-dominated eras.

However, most models of particle physics predict not only one but many coupled scalar fields and in the presence of additional interacting fields we must re-evaluate these results. The conservation of $\zeta$ relies on the perturbations being adiabatic during inflation. In the case of more than one field evolving, there is the possibility of entropy as well as adiabatic fluctuations during inflation. We assume that all our scalar fields will eventually decay during reheating and only adiabatic perturbations remain after inflation. The validity of this assumption is of course very dependent upon the reheating mechanism, but we will leave its investigation for future work.

In this paper we will illustrate the wider range of behavior possible in multiple field inflation from the simultaneous evolution of two fields. Some previous studies of inflation involving two fields, such as hybrid [7.8] or extended [9] inflation, only considered the case where one field evolves during inflation and the role of the second field is just to end inflation by a sudden phase transition, so the single field results apply. Double inflation models 10] invoke consecutive periods of inflation driven by two non-interacting fields. The density perturbation spectra produced by including an interaction term between these fields was investigated in Ref. [11]. Perturbations in models that involve two interacting scalar fields have usually been considered in the context of Brans- 
Dicke gravity 12 14 or more general scalar-tensor theories 15, 16, where the dilaton is expected to vary together with the inflaton field during inflation. However, it is the evolution of the second field rather than its coupling to the metric tensor that we wish to consider here. Only very recently have analytic results for general multiplefield inflation been presented by Sasaki and Stewart [17].

We will show how to evaluate in the slow-roll approximation the curvature perturbation at the end of inflation, using two alternative approaches corresponding to different gauge choices. In order to calculate the perturbation, we need to explicitly integrate along classical trajectories, and it turns out that it is only possible if the potential in separable in the different fields. We find that the presence of entropy fluctuations modifies the usual results for the scalar spectra produced by inflation. In particular, we show that the non-conservation of $\zeta$ far outside the horizon leads to the violation of the usual consistency relation between the ratio of tensor to scalar perturbations and the tilt of the gravitational wave spectrum.

\section{METRIC PERTURBATIONS}

We will consider linear perturbations about a spatially flat Friedmann-Robertson-Walker (FRW) metric with scale factor $a(t)$. The most general scalar and tensor metric perturbations can be written as 18 20

$$
\begin{aligned}
d s^{2}= & -(1+2 A) d t^{2}+2 B_{, i} d x^{i} d t+ \\
& a^{2}(t)\left[(1-2 \mathcal{R}) \delta_{i j}+2 E_{, i j}+h_{i j}\right] d x^{i} d x^{j},
\end{aligned}
$$

where $A, B, E$ and $\mathcal{R}$ are scalar perturbations and $h_{i j}$ is a transverse traceless tensor perturbation corresponding to gravitational waves. The perturbations can be decomposed into Fourier modes (with comoving wavenumber $k$ ) which can be treated separately in the linear approximation where they decouple.

However not all the scalar perturbations are physical degrees of freedom and to remove gauge artifacts we can define gauge invariant quantities [18 20]

$$
\begin{gathered}
\Phi \equiv A+\left(B-a^{2} \dot{E}\right) \\
\Psi \equiv \mathcal{R}-H\left(B-a^{2} \dot{E}\right),
\end{gathered}
$$

where $H=\dot{a} / a$ is the Hubble rate of expansion. Note that these are equivalent to the metric perturbations $A$ and $\mathcal{R}$ in the longitudinal gauge, where $E=B=0$. Moreover, for any perturbations whose spatial part of the stress energy tensor is diagonal, the equations of motion require $\Psi=\Phi[20$, so considering only linear perturbations the metric has just one scalar degree of freedom.

The scalar $\mathcal{R}$ is the intrinsic curvature perturbation on hypersurfaces of fixed time $t$, which transforms under a gauge transformation, $t \rightarrow t+\xi^{0}$ as $\mathcal{R} \rightarrow \mathcal{R}+H \xi^{0}$ [20].
It is convenient to evaluate the curvature perturbation on a hypersurface $\Sigma$ of constant energy density $\rho$, * corresponding to the choice of gauge $\xi^{0}=\delta \rho / \dot{\rho}$ 21]. This gives $\mathcal{R}_{\Sigma}=\mathcal{R}+H \delta \rho / \dot{\rho}$. The intrinsic three-curvature on this surface is ${ }^{(3)} R=4 \nabla^{2} \mathcal{R}_{\Sigma}$. Note that if we start from a flat hypersurface $(\mathcal{R}=0)$, we can interpret $\mathcal{R}_{\Sigma}$ as being due to a perturbation in the logarithm of the scale factor (or number of $e$-foldings $N$ ) on that hypersurface

$$
\mathcal{R}_{\Sigma}=\delta N=H \delta t
$$

where $\delta t=\delta \rho / \dot{\rho}$. In particular for inflation with a single field we can write $\delta t=\delta \phi / \dot{\phi}$, which gives the familiar result for the origin of density perturbations [6].

We will define a quantity

$$
\zeta \equiv \mathcal{R}_{\Sigma}-\frac{\nabla^{2} \Phi}{3 \dot{H}}=\Phi-\frac{H^{2}}{\dot{H}}\left(\Phi+H^{-1} \dot{\Phi}\right)
$$

written in terms of the gauge invariant metric potential, which coincides with $\mathcal{R}_{\Sigma}$ on scales far outside the horizon $(k \ll a H)$. The time-dependence of $\zeta$ on these superhorizon scales is given by

$$
\dot{\zeta} \simeq 3 H\left(\frac{\dot{p}}{\dot{\rho}}-\frac{\delta p}{\delta \rho}\right) \zeta
$$

where $\delta p$ and $\delta \rho$ are the perturbations in the pressure and energy density on spatially flat hypersurfaces. As we can see, Eq. (2.6) vanishes for adiabatic perturbations. This is the case for inflation with a single field, so we can evaluate the curvature perturbation at late times by equating it with that at horizon crossing. In fact, $\zeta$ continues to be conserved on super-horizon scales during the radiation- and dust-dominated eras, and therefore, we can directly equate the curvature perturbation when it left the horizon during inflation with that at re-entry.

The expression in Eq. (2.5) allows one to evaluate $\zeta$ once we know $\Phi$. To that end, one can integrate the coupled perturbation equations for $\Phi$ and the scalar fields in a particular gauge, see for instance Ref. [14,16]. This allows us to give an expression for $\zeta$ at the end of inflation in terms of the field fluctuations at horizon crossing. Alternatively, one could find $\zeta$ by evaluating the perturbation in the number of $e$-foldings, $\delta N$, given in Eq. (2.4),

$$
\zeta \simeq \mathcal{R}_{\Sigma}=\delta N
$$

as proposed recently by Sasaki and Stewart [17]. On spatially flat hypersurfaces the perturbed fields on superhorizon scales effectively obey the same equations of motion as the homogeneous background fields, see Eq.(3.2). Thus $\delta N$ is the difference in the number of $e$-foldings along neighboring classical trajectories in phase-space. In

\footnotetext{
*In single-field inflation, this corresponds to a comoving hypersurface.
} 
order to evaluate $\delta N$ at the end of inflation in terms of field fluctuations at horizon crossing, we have to integrate the background equations of motion, not only along a single trajectory but also along the perturbed trajectories. We will show that this does indeed yield the same results as those obtained by directly solving the evolution of the metric perturbations in the longitudinal gauge. The difference between the two approaches is just a choice of gauge.

In practice, whichever method one adopts, one needs to know not only the initial perturbation but also its integrated effect along the subsequent trajectory. In the following sections we shall show how the perturbations can be evaluated in specific models. However it is important to remember that $\zeta$ only remains a conserved quantity thereafter if we can treat the end of inflation as a transition at fixed energy density and the subsequent evolution is adiabatic. If we wish to match metric perturbations at the end of inflation, across a hypersurface of fixed energy density, we must match $\zeta$ on large scales 21. While we expect this to be true in single field models of inflation, it is a much more complicated issue in two-field inflation, since it depends upon the dynamics of reheating. Such an involved issue deserves further attention and is beyond the scope of this paper.

The attractive feature of Sasaki and Stewart's approach is that Eq. (2.7) is a purely geometrical result, independent of the matter content (subject only to the condition $\Psi=\Phi$ ) and could in principle also be applied to calculate $\zeta$ on surfaces long after the end of inflation. However evaluating $\delta N$ in the radiation-dominated era once again requires a quantitative understanding of reheating along different trajectories. In what follows we will restrict ourselves to a calculation of the curvature perturbation at the end of inflation.

\section{TWO-FIELD MODELS OF INFLATION}

In this section we will consider a model with two scalar fields, described by the action

$$
\begin{aligned}
S=\int & d^{4} x \sqrt{g}\left[\frac{1}{2 \kappa^{2}} R-\frac{1}{2} g^{a b} \phi_{, a} \phi_{, b}\right. \\
& \left.-\frac{1}{2} e^{-2 h(\phi)} g^{a b} \sigma_{, a} \sigma_{, b}-W(\phi, \sigma)\right],
\end{aligned}
$$

where $R$ is the usual Ricci curvature scalar and $\kappa^{2}=$ $8 \pi G$. If $h=0$ then the fields have standard kinetic terms, but we have also allowed for the possibility that the $\sigma$ kinetic term has a $\phi$-dependent pre-factor as would come from a conformal transformation of a theory with a nonminimally coupled $\phi$ field. Such an action might arise, for instance, in the Einstein framef of general scalartensor gravity theories [16], in which case $W(\phi, \sigma)=$ $e^{-4 h(\phi)} V(\sigma)$.

The field equations for the fields $\sigma$ and $\phi$ in a spatially flat FRW metric are then

$$
\begin{aligned}
\ddot{\sigma}+3 H \dot{\sigma} & =-e^{2 h(\phi)} \frac{\partial W}{\partial \sigma}+2 h^{\prime}(\phi) \dot{\phi} \dot{\sigma}, \\
\ddot{\phi}+3 H \dot{\phi} & =-\frac{\partial W}{\partial \phi}-h^{\prime}(\phi) e^{-2 h(\phi)} \dot{\sigma}^{2}, \\
\dot{H} & =-\frac{\kappa^{2}}{2}\left(\dot{\phi}^{2}+e^{-2 h(\phi)} \dot{\sigma}^{2}\right),
\end{aligned}
$$

and the Hamiltonian constraint is

$$
H^{2}=\frac{\kappa^{2}}{6}\left[\dot{\phi}^{2}+e^{-2 h(\phi)} \dot{\sigma}^{2}+2 W(\phi, \sigma)\right] .
$$

The condition for inflation to occur $|\dot{H}|<H^{2}$ is thus, see Eqs. (3.2) and 3.3),

$$
\dot{\phi}^{2}+e^{-2 h} \dot{\sigma}^{2}<W(\phi, \sigma)
$$

In calculating perturbations on a comoving scale $k$, we shall see that an important quantity is the number of $e$ folds from the end of inflation when that scale crossed outside the horizon $\left(k=a_{*} H_{*}\right)$,

$$
N=-\int_{e}^{*} H d t
$$

Our present horizon crossed outside the Hubble scale about 50 to $60 e$-foldings before the end of inflation. The precise number depends logarithmically on the energy scale during inflation and the efficiency of reheating, and so is weakly model-dependent.

We can derive an exact expression for the time dependence of $\zeta$ using the linearly perturbed field equations 16

$$
\dot{\zeta}=-\frac{H}{\dot{H}} \nabla^{2} \Phi+H\left(\frac{\delta \phi}{\dot{\phi}}-\frac{\delta \sigma}{\dot{\sigma}}\right) \Upsilon
$$

where

$$
\Upsilon=\frac{1}{2} \frac{d}{d t}\left(\frac{\dot{\phi}^{2}-e^{-2 h} \dot{\sigma}^{2}}{\dot{\phi}^{2}+e^{-2 h} \dot{\sigma}^{2}}\right)+h^{\prime} \dot{\phi}\left(\frac{e^{-2 h} \dot{\sigma}^{2}}{\dot{\phi}^{2}+e^{-2 h} \dot{\sigma}^{2}}\right)^{2} .
$$

Note that although $\delta \phi$ and $\delta \sigma$ are gauge dependent quantities, their combination in Eq. (3.6) is gauge invariant.

\footnotetext{
${ }^{\dagger}$ The original Jordan frame, in which $\sigma$ is minimally coupled, is related to the Einstein frame used here via the conformal transformation $\bar{g}_{a b}=e^{2 h(\phi)} g_{a b}$.
} 
If only one field is evolving ( $\dot{\sigma}=0$ or $\dot{\phi}=0$ ), we see that $\Upsilon=0$ and $\zeta$ is conserved on large scales $(k \ll a H)$, thus recovering the well known result [22]. However, if $\Upsilon \neq 0$ and both fields are evolving, $\zeta$ only remains constant for perturbations along the classical trajectory $(\delta \sigma / \delta \phi=\dot{\sigma} / \dot{\phi})$. The first term on the right-hand-side of Eq. (3.7) is present whenever two fields are evolving. The second term is due solely to the presence of the nonstandard kinetic term for $\sigma$ and vanishes when $h=$ constant. It represents the frictional damping of the $\sigma$ field by $h(\phi)$.

\section{A. Slow-roll trajectories}

To make further progress we will work in the slowroll approximation in both scalar fields. In principle this is not necessary for inflation to occur: one of the fields might roll quickly to the minimum of its potential and then the problem reduces to single field inflation. Models of hybrid inflation [7] or other models of first-order inflation [9, 8] provide examples where more than one field is present but only one field slow-rolls during inflation. However, here we wish to consider the case in which both fields slow-roll. The slow-roll approximation amounts to reducing the full field equations (3.2) to first-order equations,

$$
\begin{aligned}
3 H^{2} & \simeq \kappa^{2} W(\phi, \sigma), \\
\dot{\sigma} & \simeq-e^{2 h(\phi)} \frac{\partial \ln W}{\partial \sigma} \frac{H}{\kappa^{2}}, \\
\dot{\phi} & \simeq-\frac{\partial \ln W}{\partial \phi} \frac{H}{\kappa^{2}} .
\end{aligned}
$$

This approximation reduces the effective four-dimensional phase-space during inflation down to the twodimensional field space $\{\phi, \sigma\}$.

This leads to a crucial difference between single-field inflation and inflation with two or more fields. In single-field inflation the slow-roll solution forms a onedimensional phase-space, i.e., there is a unique trajectory. The end of inflation corresponds to a fixed value of the field and any comoving scale which crossed the horizon $N$ $e$-foldings before the end of inflation also corresponds to a unique value which may be calculated from Eq. (3.5). In two-field inflation the slow-roll approximation leaves a two-dimensional phase-space. Classical trajectories during inflation in this field space correspond to lines which are no longer unique. In particular, the end of inflation will in general be described by a one-dimensional line in this phase-space rather than a single point, as will the locus of a given number of $e$-foldings from the end of inflation.

To calculate $\zeta$ using the formalism of Sasaki and Stewart we need to know how the dependence of the number of e-foldings from the end of inflation upon the perturbations in the fields $\phi$ and $\sigma$. In the single field case this can only amount to a perturbation along the classical trajectory, $\delta t=\delta \phi / \dot{\phi}$, due to the equations of motion [6]. However, in the two-dimensional field space, perturbations will in general move the fields onto a different trajectory with a different end point of inflation, except when the perturbations happen to be adiabatic.

Therefore, it may no longer be sufficient to use the familiar relations along a given trajectory $\gamma$,

$$
\left(\frac{\partial N}{\partial \phi}\right)_{\gamma} \simeq-\frac{H}{\dot{\phi}} \quad\left(\frac{\partial N}{\partial \sigma}\right)_{\gamma} \simeq-\frac{H}{\dot{\sigma}}
$$

in order to work out the change in the number of $e$ foldings due to perturbations in the fields. To evaluate $\zeta$ at the end of inflation using Eq. (2.7), we have to allow for variations away from the classical trajectory.

Fortunately, in the case of a separable potential,

$$
W(\phi, \sigma)=U(\phi) V(\sigma),
$$

we can label the slow-roll trajectories by an integral of motion,

$$
C=\kappa^{2} \int \frac{V d \sigma}{V^{\prime}(\sigma)}-\kappa^{2} \int e^{2 h(\phi)} \frac{U d \phi}{U^{\prime}(\phi)},
$$

which allows us to parametrize motion off the classical trajectory. We can then substitute the slow-roll equation of motion for $\phi$ into Eq. (3.5) to obtain the number of $e$ foldings for a given value of $\phi_{*}$ along a trajectory labeled by $C$,

$$
N\left(\phi_{*}, \phi_{e}(C)\right) \simeq \kappa^{2} \int_{e}^{*} \frac{U d \phi}{U^{\prime}(\phi)} .
$$

This depends on the values of both $\phi_{*}$ and $\sigma_{*}$ through the dependence of $\phi_{e}$ upon $C\left(\phi_{*}, \sigma_{*}\right)$.

One should, of course, be cautious about evaluating the perturbation by integrating along a slow-roll trajectory that is only an approximation to the full equations of motion. In the single-field case the conservation of $\zeta$ is an exact result and does not rely on the slow-roll approximation. In the examples presented in this paper we have checked by numerical solutions that the slow-roll results remain a good approximation for a wide range of parameters right up until the end of inflation.

\section{B. Scalar perturbations}

Perturbations during inflation are expected to arise from the vacuum fluctuations of the fields which are stretched by the inflationary expansion up to superhorizon scales. In the following we refer to the gauge invariant scalar field perturbations [20], or equivalently the

\footnotetext{
${ }^{\ddagger}$ One can verify from the slow-roll equations of motion that $\dot{C} \simeq 0$ along any classical trajectory $\gamma$.
} 
scalar field perturbations in the longitudinal gauge. For large values of $k \gg a H$ we can neglect the potential terms in the perturbed field equations for the scalar fields and they reduce to those of massless fields. Thus, to lowest order in the slow-roll parameters, the expectation values of the perturbations as they cross outside the Hubble radius $\left(k \simeq a_{*} H_{*}\right)$ are given by Gaussian random variables with $e^{-2 h_{*}}\left\langle\left|\delta \sigma_{*}\right|^{2}\right\rangle=H_{*}^{2} / 2 k^{3}, \quad\left\langle\left|\delta \phi_{*}\right|^{2}\right\rangle=H_{*}^{2} / 2 k^{3}$, where $k$ is the comoving wavenumber. Note that, while the field $\phi$ has a standard kinetic term in Eq. (3.1), the $\sigma$ field does not and so the $\phi$-dependent prefactor must be included in the expectation value acquired at horizon crossing 23]. We shall denote the spectrum of a quantity $A$ by $\mathcal{P}_{A}(k) \equiv\left(4 \pi k^{3} /(2 \pi)^{3}\right)\left\langle|A|^{2}\right\rangle$, as defined in [2]. Thus we have

$$
\begin{aligned}
& \mathcal{P}_{\delta \phi} \simeq\left(\frac{H_{*}}{2 \pi}\right)^{2}, \\
& \mathcal{P}_{\delta \sigma} \simeq e^{2 h_{*}}\left(\frac{H_{*}}{2 \pi}\right)^{2} .
\end{aligned}
$$

For slowly varying, long-wavelength $(k \ll a H)$ modes, to lowest order in the slow-roll parameters, the perturbation equations can be integrated to give [16],

$$
\begin{aligned}
& \delta \phi \simeq \frac{1}{\kappa^{2}} \frac{U^{\prime}(\phi)}{U(\phi)} Q_{1} \\
& \delta \sigma \simeq \frac{1}{\kappa^{2}} \frac{V^{\prime}(\sigma)}{V(\sigma)}\left(Q_{2}+e^{2 h} Q_{1}\right) .
\end{aligned}
$$

where $Q_{1}$ and $Q_{2}$ are constants of integration. It will be convenient to define a new constant $Q_{3} \equiv Q_{2}+e^{2 h_{*}} Q_{1}$, so that $Q_{1}$ and $Q_{3}$ are independent Gaussian random variables whose values, for a given Fourier mode, are determined by the amplitude of $\delta \sigma_{*}$ and $\delta \phi_{*}$ at horizoncrossing. Thus they have expectation values

$$
\begin{gathered}
\mathcal{P}_{Q_{1}}=\frac{\kappa^{2}}{2 \epsilon_{\phi}^{*}}\left(\frac{H_{*}}{2 \pi}\right)^{2}, \\
\mathcal{P}_{Q_{3}}=\frac{\kappa^{2} e^{2 h_{*}}}{2 \epsilon_{\sigma}^{*}}\left(\frac{H_{*}}{2 \pi}\right)^{2} .
\end{gathered}
$$

During slow-roll, in the long wavelength limit, the curvature perturbation on hypersurfaces of constant energy density can be written as 15,24

$$
\begin{aligned}
\zeta & \simeq H \frac{\dot{\phi} \delta \phi+e^{-2 h} \dot{\sigma} \delta \sigma}{\dot{\phi}^{2}+e^{-2 h} \dot{\sigma}^{2}} \\
& \simeq \frac{\left[\epsilon_{\phi}+\left(e^{2 h}-e^{2 h_{*}}\right) \epsilon_{\sigma}\right] Q_{1}+\epsilon_{\sigma} Q_{3}}{\epsilon_{\phi}+e^{2 h} \epsilon_{\sigma}}
\end{aligned}
$$

where we have extended the usual definition of the slowroll parameter $\epsilon$ for a single field 25] to

$$
\epsilon_{\sigma} \equiv \frac{1}{2 \kappa^{2}}\left(\frac{V^{\prime}(\sigma)}{V(\sigma)}\right)^{2}, \quad \epsilon_{\phi} \equiv \frac{1}{2 \kappa^{2}}\left(\frac{U^{\prime}(\phi)}{U(\phi)}\right)^{2} .
$$

If either of the scalar fields is fixed, $\epsilon_{\sigma}$ or $\epsilon_{\phi}$ identically zero, then we recover the single field results where $\zeta$ is constant and equal to $Q_{1}$ or $Q_{3}$, respectively. We see from Eq. (3.6) that $\zeta$ continues to evolve after horizon crossing if both fields are evolving and $\Upsilon \neq 0$. In the slow-roll approximation we have

$$
\dot{\zeta} \simeq e^{2\left(h_{*}-h\right)}\left(e^{-2 h_{*}} Q_{3}-Q_{1}\right) \Upsilon .
$$

However, if $h$ grows significantly during inflation, then the change in $\zeta$ may be small, as can happen in the case of scalar-tensor gravity theories [16,13. On the other hand, for minimally coupled scalar fields, where $h=0$, we have $\Delta \zeta \simeq\left(Q_{3}-Q_{1}\right) \int \Upsilon d t$ which is typically of order $\zeta_{*}$.

The spectrum of density perturbations at the end of inflation $\mathcal{P}_{\zeta}^{e}(k)$ computed from Eq. (3.21) is,

$$
\begin{aligned}
\mathcal{P}_{\zeta}^{e} \simeq & \frac{\kappa^{2}}{2}\left(\frac{H_{*}}{2 \pi}\right)^{2}\left[\left(\frac{\epsilon_{\phi}^{e}+\left(e^{2 h_{e}}-e^{2 h_{*}}\right) \epsilon_{\sigma}^{e}}{\epsilon_{\phi}^{e}+e^{2 h_{e}} \epsilon_{\sigma}^{e}}\right)^{2} \frac{1}{\epsilon_{\phi}^{*}}\right. \\
& \left.+\left(\frac{\epsilon_{\sigma}^{e}}{\epsilon_{\phi}^{e}+e^{2 h_{e}} \epsilon_{\sigma}^{e}}\right)^{2} \frac{e^{2 h_{*}}}{\epsilon_{\sigma}^{*}}\right]
\end{aligned}
$$

where we have used Eqs. (3.19) and (3.20). This is the result found in Ref. [16.

We will now show that Eq. (3.24) can also be derived in the framework of Sasaki and Stewart [17] in terms of the change in the number of $e$-foldings, $\delta N$, as given in Eq. (2.7). We wish to evaluate $\zeta_{e}$, the curvature perturbation on a hypersurface of fixed energy density, $U\left(\phi_{e}\right) V\left(\sigma_{e}\right)=$ constant, near the end of inflation. The values of the fields $\phi_{e}$ and $\sigma_{e}$ at the end of inflation on a given classical trajectory will be a function of the conserved quantity, $C$, defined in Eq. (3.13). Therefore,

$$
\left(\frac{U^{\prime}}{U}\right)_{e} \frac{d \phi_{e}}{d C}+\left(\frac{V^{\prime}}{V}\right)_{e} \frac{d \sigma_{e}}{d C}=0 .
$$

Differentiating Eq. (3.13) with respect to the trajectory $C$, and using Eq. (3.25), we find

$$
\begin{aligned}
\kappa^{2} \frac{d \sigma_{e}}{d C} & =\left(\frac{V}{V^{\prime}}\right)_{e}\left[\left(\frac{V}{V^{\prime}}\right)_{e}^{2}+e^{2 h_{e}}\left(\frac{U}{U^{\prime}}\right)_{e}^{2}\right]^{-1}, \\
\kappa^{2} \frac{d \phi_{e}}{d C} & =-\left(\frac{U}{U^{\prime}}\right)_{e}\left[\left(\frac{V}{V^{\prime}}\right)_{e}^{2}+e^{2 h_{e}}\left(\frac{U}{U^{\prime}}\right)_{e}^{2}\right]^{-1} .
\end{aligned}
$$

We can now evaluate the dependence of the number of $e$-folds $N$, Eq. (3.14), on the initial values $\phi_{*}$ and $\sigma_{*}$,

$$
\begin{aligned}
d N= & \kappa^{2}\left(\frac{U}{U^{\prime}}\right)_{*} d \phi_{*} \\
& -\kappa^{2}\left(\frac{U}{U^{\prime}}\right)_{e} \frac{d \phi_{e}}{d C}\left[\frac{\partial C}{\partial \phi_{*}} d \phi_{*}+\frac{\partial C}{\partial \sigma_{*}} d \sigma_{*}\right] .
\end{aligned}
$$


Using Eqs. (3.26) and (3.13) we find

$$
\begin{gathered}
\left|\frac{\partial N}{\partial \phi_{*}}\right|^{2}=\frac{\kappa^{2}}{2 \epsilon_{\phi}^{*}}\left[\frac{\epsilon_{\phi}^{e}+\left(e^{2 h_{e}}-e^{2 h_{*}}\right) \epsilon_{\sigma}^{e}}{\epsilon_{\phi}^{e}+e^{2 h_{e}} \epsilon_{\sigma}^{e}}\right]^{2}, \\
\left|\frac{\partial N}{\partial \sigma_{*}}\right|^{2}=\frac{\kappa^{2}}{2 \epsilon_{\sigma}^{*}}\left[\frac{\epsilon_{\sigma}^{e}}{\epsilon_{\phi}^{e}+e^{2 h_{e}} \epsilon_{\sigma}^{e}}\right]^{2} .
\end{gathered}
$$

These are the expressions required to evaluate $\mathcal{P}_{\mathcal{C}}^{e}$ using Eq. (2.7) with $\delta \phi_{*}$ and $\delta \sigma_{*}$ given by Eqs. 3.15) and $(3.16)$,

$$
\mathcal{P}_{\zeta}^{e}=\left(\frac{H_{*}}{2 \pi}\right)^{2}\left[\left|\frac{\partial N}{\partial \phi_{*}}\right|^{2}+e^{2 h_{*}}\left|\frac{\partial N}{\partial \sigma_{*}}\right|^{2}\right] .
$$

This expression exactly coincides with that obtained in Eq. (3.24). Note that this result applies to both scalartensor theories (with $U(\phi)=\exp \{-4 h(\phi)\}$, see Ref. [16]), and for minimally coupled $(h(\phi)=0)$ two-field inflation with a separable potential in general relativity.

\section{Tensor perturbations}

In addition to the scalar curvature perturbations that give rise to density perturbations, tensor or gravitational wave perturbations $\left[h_{i j}\right.$ in Eq. (2.1)] can also be generated from quantum fluctuations during inflation [26]. These do not couple to the matter content and are determined only by the dynamics of the background metric, so the standard results for the evolution of tensor perturbations of the metric remain valid. The two independent polarizations evolve like minimally coupled massless fields with a spectrum 20,2]

$$
\mathcal{P}_{g}=8 \kappa^{2}\left(\frac{H_{*}}{2 \pi}\right)^{2} .
$$

Note that the tilt of the gravitational wave spectrum, $n_{g} \equiv d \ln \mathcal{P}_{g} / d \ln k$, is given by

$$
n_{g}=2 \frac{\dot{H}}{H^{2}} \simeq-2\left(\epsilon_{\phi}^{*}+e^{2 h_{*}} \epsilon_{\sigma}^{*}\right) .
$$

Noting the condition for inflation given in Eq. (3.4) we see that the definitive test for inflation is the presence of a gravitational wave spectrum remains $-2<n_{g}<0$ and is unaltered by the presence of more than one scalar field. The actual measurement of this slope will be exceedingly difficult. Tensor perturbations do not contribute to structure formation and in many inflationary models the observable effect of gravitational waves is completely negligible [2].

Gravitational wave perturbations can contribute to the microwave background anisotropies only on the largest scales (scales larger than the Hubble scale at lastscattering, corresponding to about $1^{\circ}$ on the sky). Their contribution relative to scalar curvature perturbations is given by the ratio [2]

$$
R \simeq \frac{3}{4} \frac{\mathcal{P}_{g}}{\mathcal{P}_{\zeta}} .
$$

The rapid decay of the gravitational wave anisotropies on smaller scales is their most distinctive signature. If we define

$$
G \equiv \frac{\epsilon_{\phi}}{\epsilon_{\sigma}}+e^{2 h}
$$

then we can write the ratio of tensor to scalar contributions as

$$
R \simeq 12 \epsilon_{\sigma}^{*} \frac{G_{e}^{2}\left(G_{*}-e^{2 h_{*}}\right)}{\left(G_{e}-e^{2 h_{*}}\right)^{2}+e^{2 h_{*}}\left(G_{*}-e^{2 h_{*}}\right)} .
$$

Together with Eq. (3.31) one can show, see Fig. 1, that

$$
R \leq 6\left|n_{g}\right| \text {. }
$$

This result was found by Polarski and Starobinsky in their model of double inflation [27] and given in Ref. 17] for general multiple-field inflation. It generalizes the usual consistency relation between $R$ and $n_{g}$ in single field inflation, where $R \simeq 6\left|n_{g}\right|$, see Ref. [28]. It shows that in principle one could tell from the spectra of metric perturbations whether more than one field is evolving during inflation.

The inequality of Eq. (3.35) becomes an equality only when $G_{*}=G_{e}$. Note that $\zeta$ in Eq. (3.21) can be written as

$$
\zeta \simeq \frac{\left(G-e^{2 h_{*}}\right) Q_{1}+Q_{3}}{G},
$$

and therefore the inequality is saturated whenever $\zeta$ remains constant after horizon crossing. As shown in Eq. (2.6) this only occurs when the perturbations are adiabatic. This is, of course, true when only one field is evolving but we can also find a class of two-field models in which $G$ is constant in the slow-roll approximation.

Even perturbations off the classical trajectory are forced to be adiabatic $(\delta p / \delta \rho=\dot{p} / \dot{\rho})$ when the pressure $p$ is a function solely of the density. In our two-field model, the pressure and energy density are given by,

$$
\begin{aligned}
& p=\frac{1}{2}\left(\dot{\phi}^{2}+e^{-2 h} \dot{\sigma}^{2}\right)-W(\phi, \sigma), \\
& \rho=\frac{1}{2}\left(\dot{\phi}^{2}+e^{-2 h} \dot{\sigma}^{2}\right)+W(\phi, \sigma),
\end{aligned}
$$

and so $p=p(\rho)$ implies that the kinetic energy density is a function of the potential. We will now show that in the slow-roll approximation for a separable potential this leads to $G$ being conserved. Along the classical trajectories,

$$
\dot{G}=\left(\frac{\epsilon_{\phi}^{\prime}}{\epsilon_{\sigma}}+2 h^{\prime} e^{2 h}\right) \dot{\phi}-\frac{\epsilon_{\phi} \epsilon_{\sigma}^{\prime}}{\epsilon_{\sigma}^{2}} \dot{\sigma}
$$


If the kinetic energy is to be a function solely of the potential energy density, we require $\epsilon_{\phi}+e^{2 h} \epsilon_{\sigma}=f(W)$, which implies that

$$
\dot{G}=\frac{\epsilon_{\phi}}{\epsilon_{\sigma}}\left(\frac{\dot{\phi}}{\epsilon_{\phi}} \frac{\partial \ln W}{\partial \phi}-\frac{\dot{\sigma}}{e^{2 h} \epsilon_{\sigma}} \frac{\partial \ln W}{\partial \sigma}\right) \frac{d f}{d \ln W},
$$

which from the slow-roll equations of motion must be zero. Thus we see that both the relation $R=6\left|n_{g}\right|$ and the constancy of $\zeta$ are a consequence of the scalar perturbations being adiabatic rather than the number of fields present.

For example, consider the class of generalized BransDicke theories [29,14] where $2 h(\phi)=\gamma \kappa \phi$ and $U(\phi)=$ $e^{-\beta \kappa \phi}$ in the Einstein frame with a polynomial inflaton potential, $V(\sigma)=\lambda \sigma^{2 n} / 2 n$. In this case, we have

$$
\epsilon_{\phi}+e^{2 h} \epsilon_{\sigma}=\frac{\beta^{2}}{2}+e^{\gamma \kappa \phi} \frac{2 n^{2}}{\kappa^{2} \sigma^{2}} .
$$

We see that for $n=\beta / \gamma$ we have $f(W)=\beta^{2} / 2+$ $2 n^{2} / \kappa^{2}(2 n W / \lambda)^{1 / n}$. As discussed above, this ensures that all perturbations are adiabatic and thus $\zeta$ is conserved. We could also see this by evaluating $G$, which in this case becomes $G=\beta \gamma C$, where $C$ is the conserved quantity along the classical trajectory, given in Eq. (3.13). Thus $\zeta$ is constant, see Eq. (3.36), and $R=6\left|n_{g}\right|$.

In particular, we find that $\zeta$ remains a constant after horizon crossing in Brans-Dicke gravity, where $\beta=2 \gamma$, for a quartic potential $V=\lambda \sigma^{4} / 4$. We will study this case in more detail in the next subsection.

\section{Brans-Dicke case}

We will study here a very simple case which we can solve completely. This is Brans-Dicke case with a quartic potential for the inflaton field, $h(\phi)=\alpha \kappa \phi, U(\phi)=$ $e^{-4 \alpha \kappa \phi}$, and $V(\sigma)=\lambda \sigma^{4} / 4$. The constant $\alpha$ characterizes the relative coupling of scalar and tensor fields to matter, and is related to the usual Brans-Dicke parameter by $2 \alpha^{2}=1 /(2 \omega+3)$ 16]. As shown in the preceding section this belongs to the sub-class of two-field models for which $\zeta$ is in fact conserved on scales outside the horizon in the slow-roll approximation.

Following Eq. (3.13), each classical trajectory can be parametrized by

$$
G=8 \alpha^{2} C=\frac{\epsilon_{\phi}}{\epsilon_{\sigma}}+e^{2 h}
$$

where $\epsilon_{\phi}=8 \alpha^{2}$ and $\epsilon_{\sigma}=8 / \kappa^{2} \sigma^{2}$ are the slow-roll parameters defined in Eq. (3.22).

The spectrum of curvature perturbations is then

$$
\begin{aligned}
\mathcal{P}_{\zeta}^{e} & \simeq \frac{\kappa^{2}}{2}\left(\frac{H_{*}}{2 \pi}\right)^{2} \frac{1}{G \epsilon_{\sigma}^{*}} \\
& \simeq \frac{\kappa^{2}}{2}\left(\frac{H_{*}}{2 \pi}\right)^{2} \frac{1}{\epsilon_{\phi}+e^{2 h_{*}} \epsilon_{\sigma}^{*}}
\end{aligned}
$$

This is an extremely simple and compact formula which makes it possible to compute the spectral tilt of the scalar perturbations, $n_{s}-1 \equiv d \ln \mathcal{P}_{\zeta}^{e} / d \ln k$,

$$
n_{s}-1=-2 \epsilon_{\phi}-3 e^{2 h_{*}} \epsilon_{\sigma}^{*}
$$

The ratio of tensor to scalar perturbations is given by

$$
R \simeq 12 G \epsilon_{\sigma}^{*} \simeq 12\left(\epsilon_{\phi}+e^{2 h_{*}} \epsilon_{\sigma}^{*}\right)
$$

Depending on $e^{2 h} \epsilon_{\sigma}$ at horizon crossing, we might have significant gravitational wave contributions. As shown earlier, the tensor perturbations' spectral index is given by $n_{g} \simeq-R / 6$, the same relation as in the single field case.

\section{E. Minimally coupled case}

If we restrict our attention to fields which are minimally coupled, i.e., $h=0$, with a separable potential, then there is no frictional damping of the $\sigma$ field and the results simplify compared with the general case. The spectrum of curvature perturbations at the end of inflation in Eq. (3.24) becomes

$$
\mathcal{P}_{\zeta}^{e} \simeq \frac{\kappa^{2}}{2}\left(\frac{H_{*}}{2 \pi}\right)^{2}\left[\left(\frac{\epsilon_{\phi}^{e}}{\epsilon_{\phi}^{e}+\epsilon_{\sigma}^{e}}\right)^{2} \frac{1}{\epsilon_{\phi}^{*}}+\left(\frac{\epsilon_{\sigma}^{e}}{\epsilon_{\phi}^{e}+\epsilon_{\sigma}^{e}}\right)^{2} \frac{1}{\epsilon_{\sigma}^{*}}\right]
$$

and the ratio between the tensor and scalar contributions to the microwave background anisotropies on large scales is given by

$$
R \simeq 12 \frac{\left(\epsilon_{\phi}^{e}+\epsilon_{\sigma}^{e}\right)^{2} \epsilon_{\phi}^{*} \epsilon_{\sigma}^{*}}{\left(\epsilon_{\phi}^{e}\right)^{2} \epsilon_{\sigma}^{*}+\left(\epsilon_{\sigma}^{e}\right)^{2} \epsilon_{\phi}^{*}} .
$$

Only if either $\epsilon_{\phi}$ or $\epsilon_{\sigma}$ are zero, or if both are constants (corresponding to exponential potentials), are all perturbations adiabatic, $\zeta$ conserved and $R=6\left|n_{g}\right|$.

More generally, the perturbations may still become effectively adiabatic by the end of inflation if the evolution has become essentially one-dimensional along, say, the $\sigma$ direction in field-space. This requires not only $\epsilon_{\sigma}^{e} \gg \epsilon_{\phi}^{e}$, but also $\zeta$ on a given scale to be due to perturbations in the $\sigma$ field, which from Eq. (3.21) requires $\epsilon_{\sigma}^{e} \gg \epsilon_{\phi}^{e}\left(Q_{1} / Q_{3}\right) \simeq \epsilon_{\phi}^{e} \sqrt{\epsilon_{\sigma}^{*} / \epsilon_{\phi}^{*}}$. In this case $\zeta$ has become constant by the end of inflation and, just as in the single field case, we expect it to remain conserved until that scale re-enters the horizon during radiation- or dustdomination. Nonetheless we may still see evidence of the second field evolving at horizon crossing during inflation due to the decrease in the Hubble rate, $H_{*}$, caused by the slow-roll of $\phi$ in addition to that due to the evolution of $\sigma$. The tilt of the scalar perturbation spectrum in this limit is 


$$
n_{s}-1 \simeq-6 \epsilon_{\sigma}^{*}+2 \eta_{\sigma}^{*}-2 \epsilon_{\phi}^{*},
$$

where we have introduced a further slow-roll parameter $\eta_{\sigma} \equiv V^{\prime \prime} / \kappa^{2} V$ which describes the curvature of the potential along $\sigma$. Only as $\epsilon_{\phi}^{*} \rightarrow 0$ do we recover the familiar single-field result [28,2]. The tilt of the spectrum of tensor perturbations is $n_{g}=-2\left(\epsilon_{\sigma}^{*}+\epsilon_{\phi}^{*}\right)$. Thus the slope of the second field tends to decrease both $n_{s}$ and $n_{g}$. However the ratio between tensor and scalar modes is given by $R \simeq 12 \epsilon_{\sigma}^{*}$ and so is a function solely of the slope of the potential along $\sigma$ at horizon crossing, as in the single field case. Thus the evolution of the $\phi$ leads to the violation of the single-field consistency relation, $R \simeq-6 n_{g}$.

\section{CONCLUSIONS}

The presence of more than one field evolving during inflation has important consequences for some of the familiar results quoted in inflationary cosmology. In the slow-roll approximation, $n$ fields lead to an $n$-dimensional phase-space. Thus, there is no longer a single classical trajectory that leads to the end of inflation and different initial conditions may lead to different end-points. Quantum fluctuations lead to perturbations not only along the trajectory but also onto neighboring trajectories. We have entropy as well as adiabatic perturbations and, in this case, the curvature perturbation $\zeta$ is no longer conserved on super-horizon scales. Therefore the perturbation on a given scale is no longer determined solely by quantities at horizon crossing, but also depends upon the subsequent evolution. In order to evaluate $\zeta$ at the end of inflation we must be able to integrate the perturbation along the trajectory. We have shown how this may be performed for a separable potential in the slow-roll approximation.

In single-field inflation the ratio between the contribution of tensor to scalar perturbations of the microwave background on large scales can be related to the tilt of the gravitational wave spectrum, $R=-6 n_{g}$ [28,5]. We have shown that this is a consequence of the scalar perturbations being adiabatic and can also occur, within the slowroll approximation, in a limited class of two-field models. More generally, in two-field models with a separable potential the ratio obeys the inequality $R \leq 6\left|n_{g}\right|$, where $n_{g}$ is given in terms of the slow-roll parameters in Eq. (3.31). While in principle this is a distinctive prediction of inflation with more than one scalar field, it is very difficult to observe. By contrast the tilt of the scalar spectrum is already constrained by observations but does not give a model-independent test of multiple-field inflation.

While we have been careful to calculate the evolution of the curvature perturbation during inflation, we have not attempted to go beyond the end of inflation. Since the observable quantity is the amplitude of scalar perturbations at re-entry during the radiation- or dust-dominated eras, the interpretation of our results is sensitive to the evolution after inflation and in particular to the dynamics of reheating. If the end of inflation corresponds to a fixed energy density then $\zeta$ will be conserved across this hypersurface. This is the case when there is a unique end-point and only one field is evolving at the end of inflation. However in the presence of two or more fields this need no longer be true and requires a more detailed study.

An important question remains as to whether, even if a given theory has many scalar fields, one should expect to see evidence in the perturbation spectra of more than one field evolving. The evolution during the final 60 e-foldings of inflation depends upon the initial conditions, and this may lead to only one field evolving at late times. For instance, in the case of chaotic inflation in some scalar-tensor gravity theories, the stochastic evolution may fix the value of the gravitational coupling, effectively leading to single field inflation [30]. To answer the question of initial conditions, one needs to understand the stochastic evolution of the coupled fields at early times 31,24, 32.

\section{ACKNOWLEDGMENTS}

The authors are grateful to Andrew Liddle, David Lyth and Ewan Stewart for useful discussions. JGB is grateful to the organizers of the Workshop on Inflation at the Aspen Center for Physics for their hospitality. JGB and DW were supported by PPARC.

[1] A. D. Linde, Particle Physics and Inflationary Cosmology, (Harwood, Chur, Switzerland, 1990).

[2] A. R. Liddle and D. H. Lyth, Phys. Rep. 231, 1 (1993).

[3] N. Vittorio, S. Matarrese and F. Lucchin, Astrophys. J. 328, 69 (1988); A. R. Liddle, D. H. Lyth and W. Sutherland, Phys. Lett. B279, 244 (1992); R. Cen, N. Y. Gnedin, L. A. Kofman and J. P. Ostriker, Astrophys. J. Lett. 399 L11 (1992); J. A. Peacock and S. J. Dodds, Mon. Not. Roy. astr. Soc. 267, 1020 (1994).

[4] K. M. Górski, et al, Astrophys. J. Lett. 430, L89 (1994).

[5] For a review see J. E. Lidsey, A. R. Liddle, E. W. Kolb, E. J. Copeland, T. Barreiro and M. Abney, "Reconstructing the Inflaton Potential - An Overview", preprint astroph/9508078 (1995).

[6] A. H. Guth and S.-Y. Pi, Phys. Rev. Lett. 49, 1110 (1982); S. W. Hawking, Phys. Lett. B115, 295 (1982); A. A. Starobinsky, Phys. Lett. B117, 175 (1982).

[7] A. D. Linde, Phys. Lett. B259, 38 (1991); Phys. Rev. D 49, 748 (1994); E. J. Copeland, A. R. Liddle, D. H. Lyth, E. D. Stewart and D. Wands, Phys. Rev. D 49, 6410 (1994).

[8] A. D. Linde, Phys. Lett. B249, 18 (1990); F. C. Adams and K. Freese, Phys. Rev. D 43, 353 (1991). 
[9] D. La and P.J. Steinhardt, Phys. Rev. Lett. 62, 376 (1989); P.J. Steinhardt and F.S. Accetta, Phys. Rev. Lett. 64, 2470 (1990); J.D. Barrow and K. Maeda, Nucl. Phys. B341, 294 (1990); J. García-Bellido and M. Quirós, Phys. Lett. B243, 45 (1990).

[10] A. A. Starobinsky, JETP Lett. 42, 152 (1985); J. Silk and M. S. Turner, Phys. Rev. D 35, 419 (1987); D. Polarski and A. A. Starobinsky, Nucl. Phys. B385, 623 (1992); R. Holman, E. Kolb, S. L. Vadas and Y. Wang, Phys. Lett. B269, 252 (1991).

[11] L. A. Kofman and A. D. Linde, Nucl. Phys. B282, 555 (1987); L. A. Kofman and D. Yu. Pogosyan, Phys. Lett. B214, 508 (1988); D. S. Salopek, J. R. Bond and J. M. Bardeen, Phys. Rev. D 40, 1753 (1989).

[12] A. D. Linde, Phys. Lett. B238, 160 (1990).

[13] N. Deruelle, C. Gundlach and D. Langlois, Phys. Rev D46, 5337 (1992).

[14] A. A. Starobinsky and J. Yokoyama, Density fluctuations in Brans-Dicke inflation, astro-ph/9502002 (1995).

[15] S. Mollerach and S. Matarrese, Phys. Rev. D45, 1961 (1992).

[16] J. García-Bellido and D. Wands, Constraints from inflation on scalar-tensor theories, preprint SUSSEX-AST95/6-3, gr-qc/9506050. Phys. Rev. D, to appear (1995).

[17] M. Sasaki and E. D. Stewart, A general analytic formula for the spectral index, preprint astro-ph/9507001 (1995).

[18] J. M. Bardeen, Phys. Rev. D 22, 1882 (1980); J. M. Bardeen, P. J. Steinhardt and M. S. Turner, Phys. Rev. D28, 679 (1983).

[19] H. Kodama and M. Sasaki, Prog. Theor. Phys. Supp. 78, 1 (1984); M. Sasaki, Prog. Theor. Phys. 76, 1036 (1986).

[20] V. F. Mukhanov, H. A. Feldman and R. H. Brandenberger, Phys. Rep. 215, 203 (1992).

[21] N. Deruelle and V. Mukhanov, On matching conditions for cosmological perturbations, preprint gr-qc/9503050 (1995).

[22] D. H. Lyth, Phys. Rev. D31, 1792 (1985).

[23] J. García-Bellido, Nucl. Phys. B423, 221 (1994).

[24] J. García-Bellido, A. D. Linde and D. A. Linde, Phys. Rev. D50, 730 (1994).

[25] A. R. Liddle, P. Parsons and J. D. Barrow, Phys. Rev. D50, 7222 (1994).

[26] L.F. Abbott and M.B. Wise, Nucl. Phys. B244, 541 (1984).

[27] D. Polarski and A. A. Starobinsky, Phys. Lett. B356, 196 (1995).

[28] A. R. Liddle and D. H. Lyth, Phys. Lett. B 291, 391 (1992).

[29] T. Damour, G. Gibbons and C. Gundlach, Phys. Rev. Lett. 64, 123 (1990); Phys. Rev. D43, 3873 (1991).

[30] J. García-Bellido and D. Wands, General relativity as an attractor of scalar-tensor stochastic inflation, preprint SUSSEX-AST-95/3-1, gr-qc/9503049, Phys. Rev. D, to appear (1995).

[31] A. D. Linde, D. A. Linde and A. Mezhlumian, Phys. Rev. D 49, 1783 (1994).

[32] J. García-Bellido and A. D. Linde, Phys. Rev. D 51, 429 (1995); J. García-Bellido and A. D. Linde, Stationary solutions in Brans-Dicke stochastic inflationary cosmology, preprint SU-ITP-95/8, gr-qc/9504022, Phys. Rev. D, to appear (1995).

\section{FIGURE CAPTIONS}

Fig. 1. Plot of the ratio $R / 6\left|n_{g}\right|$ from Eqs. (3.31) and (3.34) as a function of the parameters $a \equiv G_{e} e^{-2 h_{*}}$ 1 and $b \equiv G_{*} e^{-2 h_{*}}-1=\epsilon_{\phi}^{*} / \epsilon_{\sigma}^{*}$. This ratio reaches a maximum of one for $a=b$, corresponding to $G_{e}=G_{*}$. In the minimally coupled case $(h=0)$, we have $a=\epsilon_{\phi}^{e} / \epsilon_{\sigma}^{e}$, so both $a$ and $b$ must be non-negative. 


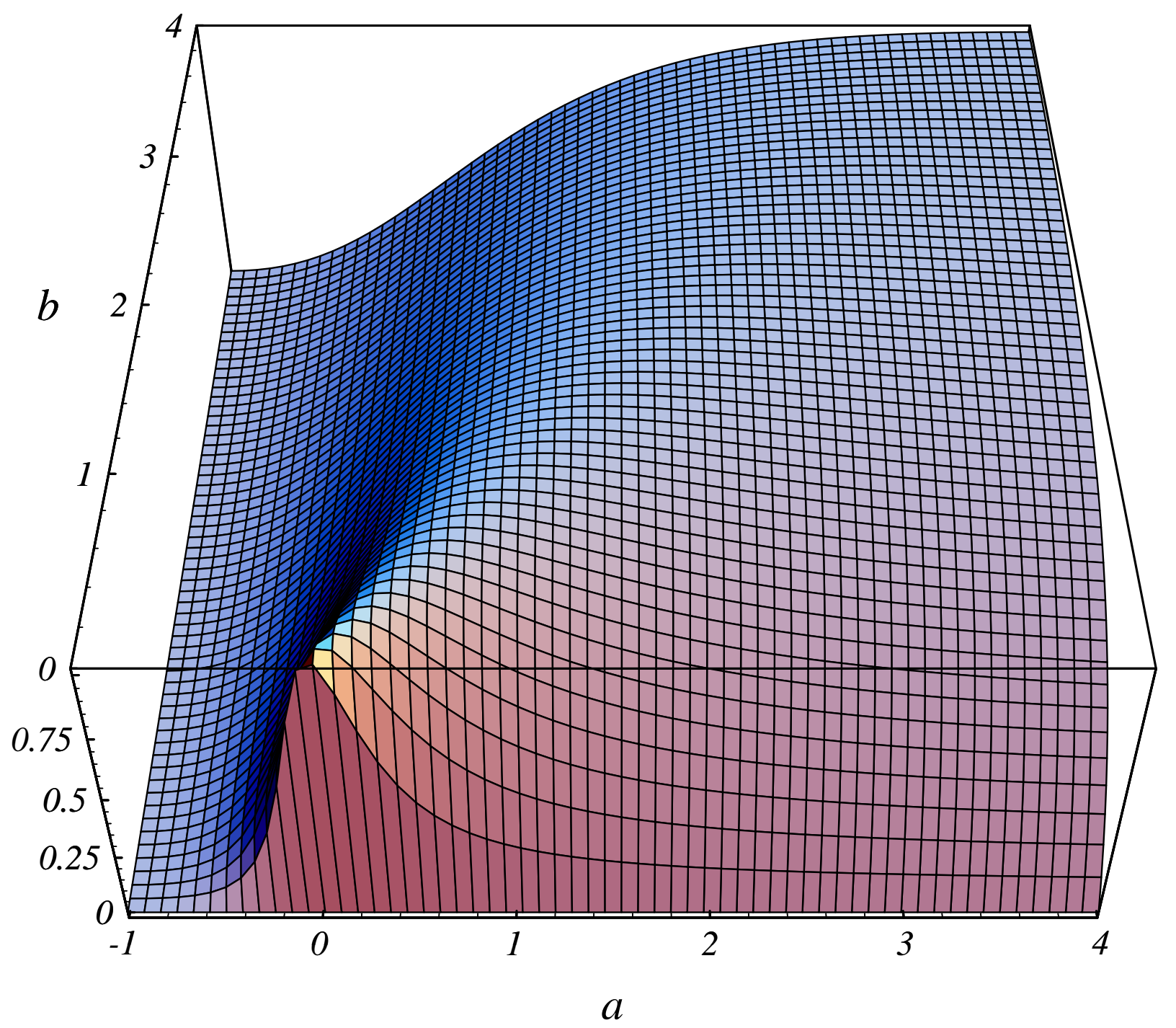

